\title{
Multiple blood parameters may serve as a warning to immunochemotherapy-related interstitial lung disease in B-cell lymphoma
}

\author{
Fan Xia ${ }^{1 \#}$, Hailing Liu ${ }^{2 \#}$, Haizhou Zhang ${ }^{1 \#}$, Nana Ping ${ }^{3}$, Peng Wang ${ }^{3}$, Zhengming Jin ${ }^{3}$, Jianguo Zhu ${ }^{1}$, \\ Changju $Q \mathbf{u}^{3}$
}

${ }^{1}$ Department of Pharmacy, The First Affiliated Hospital of Soochow University, Suzhou, China; ${ }^{2}$ Department of Radiology, Binhai County People's Hospital, Yancheng, China; ${ }^{3}$ Department of Hematology, Collaborative Innovation Center of Hematology, Institute of Blood and Marrow Transplantation, The First Affiliated Hospital of Soochow University, Jiangsu Institute of Hematology, Suzhou, China

Contributions: (I) Conception and design: F Xia, C Qu; (II) Administrative support: Z Jin, J Zhu; (III) Provision of study materials or patients: C Qu, Z Jin; (IV) Collection and assembly of data: F Xia, H Liu, H Zhang; (V) Data analysis and interpretation: N Ping, P Wang; (VI) Manuscript writing: All authors; (VII) Final approval of manuscript: All authors.

\#These authors contributed equally to this work.

Correspondence to: Zhengming Jin; Changju Qu. Department of Hematology, Collaborative Innovation Center of Hematology, Institute of Blood and Marrow Transplantation, The First Affiliated Hospital of Soochow University, Jiangsu Institute of Hematology, Suzhou 215000, China. Email: jinzhengming519519@163.com; qcj310@suda.edu.cn; Jianguo Zhu. Department of Pharmacy, The First Affiliated Hospital of Soochow University, Suzhou 215000, China. Email: 15950005195@163.com.

Background The main aim of this study was to determine some simple but meaningful parameters that indicate immunochemotherapy-related interstitial lung disease (ILD) early in B-cell lymphoma and provide direction to hematologists.

Methods: The clinical and laboratory characteristics, the treatments and outcomes of 21 B-cell lymphoma patients with ILD who underwent rituximab (RTX) -based immunochemotherapy were collected and retrospectively analyzed.

Results: More cycles of immunochemotherapy and higher cumulative doses of RTX and doxorubicin hydrochloride liposome conferred a high risk of ILD. Compared to the baseline, patients had a significantly lower white blood cell count (WBC), absolute lymphocyte count (ALC), and albumin level $\left(4.95 \times 10^{9} v s\right.$. $6.32 \times 10^{9}, 0.71 \times 10^{9}$ vs. $1.61 \times 10^{9}, 34.1$ vs. $\left.40.4 \mathrm{~g} / \mathrm{L} ; \mathrm{P}<0.05\right)$, and higher C-reactive protein (CRP), alphahydroxybutyrate dehydrogenase $(\alpha-\mathrm{HBDH})$, and lactate dehydrogenase $(\mathrm{LDH})(15.36$ vs. $7.00 \mathrm{mg} / \mathrm{L}, 293.0$ vs. $163.1 \mathrm{U} / \mathrm{L}, 361.8$ vs. $231.1 \mathrm{U} / \mathrm{L} ; \mathrm{P}<0.05)$ levels at ILD onset. Further, a positive correlation was found between early glucocorticosteroid intervention and the good prognostication of ILD. In addition, an analysis of the prognoses of 2 cases of patients with pneumocystis pneumonia (PCP) infection indicated that after 3 cycles of treatment, patients, especially unfit patients or those who have received ILD glucocorticoid treatment, may need to receive trimethoprim/sulfamethoxazole (TMP/SMX) to prevent PCP.

Conclusions: There was a relationship between variations of blood parameters and the occurrence of ILD which might serve as a warning for B-cell lymphoma patients with immunochemotherapy-related ILD.

Keywords: Blood parameters; interstitial lung disease (ILD); rituximab (RTX); immunochemotherapy; B-cell lymphoma

Submitted Jul 02, 2021. Accepted for publication Aug 26, 2021.

doi: 10.21037/apm-21-2027

View this article at: https://dx.doi.org/10.21037/apm-21-2027 


\section{Introduction}

B-cell lymphoma is a group of highly heterogeneous diseases with variable outcomes, and represents the most common type of non-Hodgkin's lymphoma (1). Since the first use of the anti-CD20 monoclonal antibody rituximab (RTX) clinically, the prognosis of patients with B-cell lymphoma has improved significantly (2-4). The RTXbased immunochemotherapy regimen is now a first-line therapy for most types of B-cell lymphoma (5). Following the increased usage of RTX, a number of rare adverse events, including interstitial lung disease (ILD), have begun to be reported more and more frequently $(6,7)$. At present, the mechanism by which RTX leads to ILD is still unknown. A potential explanation for RTX induced ILD may be the induction and release of cytotoxic substances $(8,9)$. In addition, several recent studies have shown that the use of pegylated liposomal doxorubicin in chemotherapy regimens could also cause ILD $(6,10,11)$.

Other than ILD, which is the most common type of lung disease of RTX-based immunochemotherapy, there are other types of lung disease, including alveolarinterstitial pneumonia, pulmonary fibrosis, hypersensitivity pneumonitis, and bronchiolitis obliterans organizing pneumonia (12-14). Infectious pneumonia is another common complication associated with RTX-induced pulmonary disease $(15,16)$. Infectious pneumonia, especially pneumocystis pneumonia (PCP), has a high mortality rate $(15,16)$. However, the discovery, diagnosis, and treatment of ILD during RTX-based immunochemotherapy still face a number of challenges. First, the clinical symptoms of ILD are nonspecific. Generally, fever, cough, dyspnea, and chest tightness are the most common clinical symptoms of early stage RTX-based immunochemotherapy-related ILD, and it is difficult to distinguish between ILD and other lung diseases caused by drugs, infection, tumor infiltration, or other factors $(7,17,18)$. Second, radiologic changes are delayed, and the biomarkers for RTX-based immunochemotherapy-related ILD are not yet known (19-21). Recently, Chen et al. found that the platelet/ lymphocyte ratio (PLR), which is a simple, credible, and inexpensive biomarker, is associated with an increased incidence of ILD in patients with rheumatoid arthritis (22). In addition, Gao et al. reported that the monocyte to lymphocyte ratio (MLR) could be used as a predictor of radiation pneumonia in patients undergoing thoracic tumor radiotherapy (23). However, it is not clear whether these biomarkers or other blood parameters are related to the incidence of immunochemotherapy-related ILD in patients with B-cell lymphoma.

Lymphopenia is a common side effect of RTX treatment (24). Reckzeh et al. observed that severe lymphopenia is associated with the occurrence of interstitial pneumonia in patients with lung cancer (25). Additionally, a low absolute lymphocyte count (ALC) and the addition of RTX at diagnosis was found to increase the incidence of interstitial pneumonia in patients with diffuse large B-cell lymphoma (DLBCL) (26). These reports suggest that lymphopenia may be a risk factor of ILD during RTX-based immunochemotherapy, as it changes patients' immune status.

In this study, we retrospectively collected and analyzed data on the clinical and laboratory characteristics, and treatments and outcomes of 21 B-cell lymphoma patients with ILD undergoing RTX-based immunochemotherapy. To explore some simple but meaningful parameters that indicate immunochemotherapy-related ILD early in B-cell lymphoma and provide direction to hematologists, we also analyzed the relationship between ILD and variations in laboratory data related to inflammation, immunity, and tumor status. We present the following article in accordance with the STROBE reporting checklist (available at https:// dx.doi.org/10.21037/apm-21-2027).

\section{Methods}

\section{Patient population}

Twenty-one B-cell lymphoma patients who developed ILD while undergoing immunochemotherapy administered at the First Affiliated Hospital of Soochow University from March 2014 to November 2020 were enrolled in this study. To be eligible to participate in this study, patients had to meet the following inclusion criteria: (I) have been pathologically diagnosed with B-cell lymphoma and have received RTX-based immunochemotherapy at our center; (II) be aged $\geq 18$ years old; (III) have computed tomography (CT) scans that showed no obvious ILD and no lymphoma infiltration before the first treatment; (IV) have a diagnosis of ILD that met typical radiologic changes, have respiratory symptoms, and have a history of immunochemotherapy. Patients were excluded from the study if they did not have CT scans at the baseline, midpoint, or at the time at which symptoms appeared. The study was conducted in accordance with the Declaration of Helsinki (as revised in 2013). The study was approved by the Ethics Committee of 
Table 1 Patients' demographic and clinical characteristics

\begin{tabular}{|c|c|}
\hline Variables & Numbers (percentage) \\
\hline \multicolumn{2}{|l|}{ Gender } \\
\hline Female & $12(57.1)$ \\
\hline Male & $9(42.9)$ \\
\hline Median age (year) ${ }^{\star}$ & $53[46-60]$ \\
\hline \multicolumn{2}{|l|}{ B-cell lymphoma type } \\
\hline DLBCL & $18(85.7)$ \\
\hline $\mathrm{FL}$ & $2(9.5)$ \\
\hline MZL & $1(4.8)$ \\
\hline \multicolumn{2}{|l|}{ Stage } \\
\hline I-II & $10(47.6)$ \\
\hline III-IV & $11(52.4)$ \\
\hline B symptoms & $4(19.0)$ \\
\hline $\mathrm{Ki}-67 \geq 90 \%$ & 7 (33.3) \\
\hline LDH elevated & 9 (42.9) \\
\hline \multicolumn{2}{|l|}{ IPI } \\
\hline $0-1$ & $11(52.4)$ \\
\hline $2-5$ & $10(47.6)$ \\
\hline \multicolumn{2}{|l|}{ Type of immunochemotherapy } \\
\hline $\mathrm{R}-\mathrm{CDOP}$ & $16(76.2)$ \\
\hline R-CHOP/R-CHOPE/R-EPOCH & $5(23.8)$ \\
\hline
\end{tabular}

*, values are expressed as median [inter-quartile range]. DLBCL, diffuse large B-cell lymphoma; FL, follicular lymphoma; MZL, marginal zone lymphoma; LDH, lactose dehydrogenase; IPI, international prognostic index.

the First Affiliated Hospital of Soochow University (Suzhou, China). Individual consent for this retrospective analysis was waived.

\section{Immunochemotberapy regimen}

In addition to RTX, all the patients received one of the following chemotherapy regimens: (I) CDOP: cyclophosphamide, doxorubicin hydrochloride liposome, vindesine, and dexamethasone; (II) CHOP: cyclophosphamide, doxorubicin/epirubicin, vindesine, and dexamethasone/prednisone; (III) EPOCH: cyclophosphamide, doxorubicin, vindesine, etoposide and dexamethasone; and (IV) CHOPE: cyclophosphamide, doxorubicin, vindesine, etoposide and dexamethasone.

\section{Immunocbemotberapy-related ILD}

Immunochemotherapy-related ILD was diagnosed on the basis of: temporal relationship between exposure to RTX-based immunochemotherapy and the development of respiratory symptoms, including fever, cough, chest congestion, or dyspnea; chest CT scans showed interstitial pulmonary infection; and exclusion of other known causes of ILD.

\section{Blood parameters}

Blood biochemical indexes including the C-reactive protein (CRP), albumin, alpha-hydroxybutyrate dehydrogenase $(\alpha-\mathrm{HBDH})$ and lactate dehydrogenase (LDH) were acquired by automatic biochem analyzer of ADVIA 2400 of SIEMENS (Germany) and blood routin indexes including platelet, white blood cell count (WBC), neutrophil, ALC and monocyte were tested by Sysmex XN-9000 Automatic Hematology Analyzer (Japan). All blood parameters were detected using peripheral venous blood of patients after overnight fasting.

\section{Statistical analysis}

The statistical analyses were performed using SPSS version 20.0 software. The categorical variables are presented as the frequency and percentage, and the continuous variables were analyzed using descriptive statistical methods (median and range) and Mann-Whitney $\mathrm{U}$ tests. A $\mathrm{P} \leq 0.05$ was considered statistically significant.

\section{Results}

\section{Patient characteristics}

The demographic and clinical characteristics of the 21 patients are set out in Table 1. The cohort comprised 12 females and 9 males. Patients' had a median age of 53 [46-60] years. Most patients $(18 / 21,85.7 \%)$ had a diagnosis of DLBCL and more than half $(11 / 21,52.4 \%)$ were at an advanced stage (stage III-IV). Nineteen percent (4/21) of patients presented with B symptoms, 33.3\% (7/21) had a higher Ki-67 ( $\geq 90 \%), 42.9 \%(9 / 21)$ had elevated LDH at diagnosis, and $47.6 \%(10 / 21)$ had an international prognostic index $(\mathrm{IPI}) \geq 2$. Most patients received immunochemotherapy with R-CDOP (16/21, 76.2\%); however, some received R-CHOP/R-CHOPE/R-EPOCH (5/21, 23.8\%) (see Table 1). 
Table 2 Characteristics of immunochemotherapy, symptoms, and CT findings of 21 patients

\begin{tabular}{|c|c|}
\hline Characteristics & Numbers (percentage) \\
\hline $\begin{array}{l}\text { Median cycles of immunochemotherapy } \\
\text { before lung disease* }\end{array}$ & $3[3-4]$ \\
\hline \multicolumn{2}{|l|}{$\begin{array}{l}\text { Cycles of immunochemotherapy before } \\
\text { lung disease }\end{array}$} \\
\hline $1-2$ cycles & $3(14.3)$ \\
\hline 3-4 cycles & $16(76.2)$ \\
\hline 5 cycles & $2(9.5)$ \\
\hline \multicolumn{2}{|l|}{$\begin{array}{l}\text { Cumulative dose of RTX before lung } \\
\text { disease }(\mathrm{mg})\end{array}$} \\
\hline$\leq 1,000$ & $1(4.8)$ \\
\hline $1,000-3,000$ & $17(80.9)$ \\
\hline$\geq 3,000$ & $3(14.3)$ \\
\hline \multicolumn{2}{|l|}{ Anthracycline chemotherapy drugs } \\
\hline Doxorubicin/epirubicin & $5(23.8)$ \\
\hline Doxorubicin hydrochloride liposome & $16(76.2)$ \\
\hline \multicolumn{2}{|l|}{ Presenting symptoms } \\
\hline Fever & $16(76.2)$ \\
\hline Cough & $16(76.2)$ \\
\hline Chest tightness & $11(52.4)$ \\
\hline Dyspnea & $5(23.8)$ \\
\hline \multicolumn{2}{|l|}{ CT findings } \\
\hline Diffuse ground-glass opacities & $14(66.7)$ \\
\hline Patchy ground-glass opacities & 7 (33.3) \\
\hline Small amount of pleural effusion & $2(9.5)$ \\
\hline
\end{tabular}

${ }^{*}$, values are expressed as median [inter-quartile range]. CT, computed tomography; RTX, rituximab.

\section{Immunochemotherapy, symptoms, and CT findings}

Patients had received a median of 3 cycles of immunochemotherapy at the onset of ILD (range: 3-4). In addition, $3(14.3 \%), 16(76.2 \%)$, and $2(9.5 \%)$ patients received $1-2,3-4$ and 5 cycles before ILD happened, respectively. Seventeen $(80.9 \%)$ patients had a cumulative dose of RTX between 1,000 and 3,000 mg, 3 (14.3\%) had $\geq 3,000 \mathrm{mg}$, and $1(4.8 \%)$ had $\leq 1,000 \mathrm{mg}$. Most patients chose doxorubicin hydrochloride liposome (16/21, 76.2\%); however, some patients chose doxorubicin or epirubicin $(5 / 21,23.8 \%)$.

The most common clinical symptoms were fever $(16 / 21$,
$76.2 \%)$ and cough $(16 / 21,76.2 \%)$. Other symptoms included chest tightness $(11 / 21,52.4 \%)$ and dyspnea $(5 / 21$, $23.8 \%)$. All patients had diffuse $(14 / 21,66.7 \%)$ or patchy $(7 / 21,33.3 \%)$ ground-glass opacities, and most patients had uniformly diffuse opacities throughout both lungs. Other radiographic findings included increased lung markings, interlobular septal thickening and consolidation, and a small amount of pleural effusion $(2 / 21,9.5 \%)$ (see Table 2$)$.

\section{Variations of blood parameters}

Table 3 shows changes in some simple blood parameters before the first immunochemotherapy and at the onset of ILD based on CT findings. The results showed that the median WBC, ALC and albumin levels were significantly lower in patients with ILD $(\mathrm{P}<0.05)$ compared to those at the baseline before immunochemotherapy. However, the CRP, $\alpha-\mathrm{HBDH}$, and LDH levels increased with the occurrence of ILD $(\mathrm{P}<0.05)$. Additionally, a higher the PLR and neutrophil/lymphocyte ratio (NLR) and lower lymphocyte/monocyte ratio (LMR) were also observed at the time of ILD onset, and there were statistically significant differences in the levels of PLR, LMR, and NLR between the baseline and ILD $(\mathrm{P}<0.05)$.

\section{Treatments and outcomes}

Patients $(15 / 21,71.4 \%)$ were treated with glucocorticosteroids. Most patients used methylprednisolone (14/15, 93.3\%) after CT findings of ILD. The initial dose of methylprednisolone was $80 \mathrm{mg} / \mathrm{d}$, and the median time was 4 days. All patients received anti-infection therapy, and $9(42.9 \%)$ patients used trimethoprim/sulfamethoxazole (TMP/SMX). Twenty (95.2\%) patients improved; however, 1 patient died of acute respiratory distress syndrome (ARDS). Among the 15 patients who were treated with glucocorticosteroids, 10 (66.7\%) displayed clinical symptom relief within 1 week, and 12 (80.0\%) displayed image remission within 2 weeks. However, of the remaining 6 patients who did not receive glucocorticosteroid therapy, only 1 patient $(16.7 \%)$ experienced clinical symptom relief within 1 week and none of the patients displayed image remission within 2 weeks (see Table 4).

\section{Discussion}

We found ILD occurred in most patients after the 3rd or 4th cycle of RTX-based immunochemotherapy. Higher cumulative doses of RTX and doxorubicin hydrochloride 
Table 3 Comparison of blood parameters between baseline and ILD onset of 21 patients

\begin{tabular}{|c|c|c|c|}
\hline Laboratory data & Baseline, median (range) & ILD at CT finding, median (range) & $\mathrm{P}$ \\
\hline WBC $\left(10^{9} / \mathrm{L}\right)$ & $6.32(4.72-8.61)$ & $4.95(3.80-6.09)$ & 0.013 \\
\hline Neutrophil $\left(10^{9} / \mathrm{L}\right)$ & $3.59(2.80-4.52)$ & $3.43(2.13-4.36)$ & 0.414 \\
\hline $\operatorname{ALC}\left(10^{9} / L\right)$ & $1.61(1.21-2.85)$ & $0.71(0.48-0.93)$ & 0.000 \\
\hline PLR & $119.57(62.09-160.33)$ & $302.13(228.92-487.67)$ & 0.000 \\
\hline LMR & $4.63(2.09-8.43)$ & $1.22(0.83-2.73)$ & 0.001 \\
\hline NLR & $2.49(1.24-2.78)$ & $5.23(3.03-7.58)$ & 0.000 \\
\hline $\mathrm{CRP}$ (mg/L) & $7.00(1.82-15.34)$ & $15.36(10.82-26.21)$ & 0.004 \\
\hline LDH (U/L) & $231.1(172.1-328.0)$ & $361.8(271.3-433.8)$ & 0.009 \\
\hline
\end{tabular}

ILD, interstitial lung disease; CT, computed tomography; WBC, white blood cell count; ALC, absolute lymphocyte count; PLR, platelet/ lymphocyte ratio; LMR, Iymphocyte/monocyte ratio; NLR, neutrophil/lymphocyte ratio; CRP, C-reactive protein; $\alpha$-HBDH, alphahydroxybutyrate dehydrogenase; $\mathrm{LDH}$, lactate dehydrogenase.

liposome conferred a high risk of ILD, and the application of glucocorticosteroid was a key therapy in the improvement of clinical symptoms and the radiologic presentation of the patients. Notably, we first comprehensively reported the variations of the multiple hematological parameters involved in inflammation, immunity and tumor status during immunochemotherapy-related ILD onset in patients with B-cell lymphoma. All these parameters are simple, inexpensive, and easily available in patients' daily routine tests.

At present, various biomarkers have been reported in the diagnosis and prognostication of lung disease, such as the serum levels of Krebs von den Lungen-6 (KL-6), thymus and activation-regulated chemokine (TARC)/CC chemokine ligand 17 (CCL17), and the level of surfactant protein-D (19-21). However, these biomarkers are either unspecific, costly, complicated to obtain, or time consuming to obtain. Thus, comparable biomarkers need to be identified urgently. Further, if biomarkers are identified that are obtained as part of patients' daily routine tests, they could be used to identify early signs of the occurrence of ILD during immunochemotherapy.

In our study, we found that the median WBC and ALC were significantly lower in patients at ILD onset compared to the baseline $\left(4.95 \times 10^{9}\right.$ vs. $6.32 \times 10^{9} ; 0.71 \times 10^{9}$ vs. $1.61 \times 10^{9}$; $\mathrm{P}<0.05)$. The decrease of ALC, called lymphopenia, is a common complication that occurs in $40 \%$ of patients treated with RTX (24). As a CD20 antibody, RTX could induce B-lymphocyte apoptosis, complement activation, and cause the release of tumor necrosis factor $\alpha$, cytokine, which may be related to the underlying mechanism of ILD (26). In addition, while there have been quite a few reports on PLR, $\mathrm{LNR}$, and LMR/MLR in the prediction of lung disease in patients with rheumatoid arthritis and thoracic tumors, little research has been conducted in this area in relation to B-cell lymphoma $(22,23)$. In our study, we found significant changes in these parameters at ILD onset compared to the baseline $(\mathrm{P}<0.05)$. We believed that the reason for these changes was mainly due to the decrease in lymphocytes.

CRP has previously been recognized as an acute phase protein that is elevated during inflammatory conditions, such as rheumatoid arthritis and infections (27). Recently, several studies have shown that increases in CRP are correlated with pulmonary complications. $\mathrm{Xu}$ et al. reported that elevated CRP was more frequent in patients with ILD caused by clinically amyopathic dermatomyositis (28). However, the relationship between CRP and ILD in patients undergoing RTX-based immunochemotherapy is still not clear. In this study, we similarly found an obviously elevated CRP at the time of ILD onset compared to the baseline in the B-cell lymphoma patients (15.36 vs. $7.00 \mathrm{mg} / \mathrm{L} ; \mathrm{P}<0.05)$. Additionally, we also observed 
Table 4 Treatments and outcomes of the 21 patients

\begin{tabular}{lc}
\hline Treatment and outcome & $\begin{array}{c}\text { Numbers } \\
\text { (percentage) }\end{array}$ \\
\hline Glucocorticosteroid usage & $15(71.4)$ \\
Methylprednisolone & $14(93.3)$ \\
Dexamethasone & $1(6.7)$ \\
Initial dose of methylprednisolone, mg/d & \\
80 mg/d & $13(92.9)$ \\
40 mg/d & $1(7.1)$ \\
Days for 80 mg/d of methylprednisolone* & $4.0(2.8-5.3)$ \\
Anti-infection usage & $21(100.0)$ \\
TMP/SMX usage & $9(42.9)$ \\
Outcomes of lung injury & \\
Improved & $20(95.2)$ \\
Dead & $1(4.8)$ \\
Efficacy of glucocorticosteroid & $12(80.0)$ \\
Clinical symptom relief within 1 week & \\
Image remission within 2 weeks & $10(66.7)$ \\
Outcome of patients who did not receive & \\
Imageocorticosteroids & \\
\hline & \\
Clinical symptom relief within 1 week & $(0.0)$ \\
\hline
\end{tabular}

*, all values are expressed as median (inter-quartile range). TMP/ SMX, trimethoprim/sulfamethoxazole

a significant decrease of albumin at the onset of ILD when ILD occurred (34.1 vs. $40.4 \mathrm{~g} / \mathrm{L} ; \mathrm{P}<0.05)$. Usually, the serum level of albumin is considered a biomarker for malnutrition, and it also plays an important role in inflammation $(29,30)$. In the inflammatory state, microvascular permeability is increased, and it leads to the alteration of albumin distribution between intravascular and extravascular compartments, which in turn leads to a decrease in serum albumin (31). We also found that two other parameters (i.e., $\alpha-\mathrm{HBDH}$ and $\mathrm{LDH}$ ) were higher at ILD onset (293.0 vs. $163.1 \mathrm{U} / \mathrm{L} ; 361.8$ vs. $231.1 \mathrm{U} / \mathrm{L}$; $\mathrm{P}<0.05)$. Research has shown that $\alpha-\mathrm{HBDH}$ is involved in different diseases, such as COVID-19 and liver injury $(32,33)$. Our study was the first to show that $\alpha-\mathrm{HBDH}$ may also be related to the occurrence of immunochemotherapyrelated ILD. LDH is not only an indicator of tumor burden in lymphoma, it is also a marker of immune suppression in cancer (34). In this study, our findings that LDH is significantly elevated at ILD onset provide further evidence that the suppression of the immune response might cause ILD.

As ILD is a common type of lung disease associated with RTX-based immunochemotherapy, the discontinuation of RTX and doxorubicin hydrochloride liposome, and the immediate administration of glucocorticoids are the main treatments. Generally, patients with a moderate ILD should be treated at a dose which is equivalent to $0.5-1.0 \mathrm{mg} / \mathrm{kg} / \mathrm{day}$ of prednisolone for $2-4$ weeks and then gradually tapered. For patients with a severe ILD, an initial dose of 500-1,000 mg/day of methylprednisolone for 3 days is recommended, and then followed the treatment above (35). Usually, symptomatic recovery is achieved within days and radiological remission within weeks to months in most patients treated with glucocorticoids (17). In our study, 15 patients (71.4\%) received glucocorticoid therapy in combination with anti-infection therapy, and 6 received anti-infection therapy only. Ultimately, 20 patients recovered, but 1 patient died immediately. Among the 16 patients who received glucocorticoid therapy, 10 experienced clinical symptom relief within 1 week, and 12 showed image remission within 2 weeks. Among the 6 patients who did not receive glucocorticosteroid therapy, only 1 patient experienced clinical symptom relief within 1 week, and none of the patients showed image remission within 2 weeks. These results led us to consider whether glucocorticosteroids should be preemptively administered to B-cell lymphoma patients with ILD during immunochemotherapy.

In addition, typical CT scans, showing bilateral pulmonary ground-glass opacities, reticular shadow and crazy paving sign, and bronchoalveolar lavage fluid NGS, confirmed that two patients had been infected with PCP (36). These findings support those of previous reports of high PCP infection rates among patients with lymphoma undergoing immunochemotherapy $(15,37)$. In our study, both patients who suffered PCP also had ILD previously during immunochemotherapy and improved or recovered after glucocorticoid administration. Case $1 \mathrm{did}$ not receive TMP/SMX until the appearance of severe hypoxemia, and unfortunately died of ARDS. Case 2 received a previously TMP/SMX treatment before their symptoms worsened and bronchoalveolar lavage fluid NGS confirmed PCP infection. Case 2 ultimately recovered (see Figure 1). Our data suggest that it is crucial to consider PCP infection during ILD onset. PCP may be able to be prevented via the 

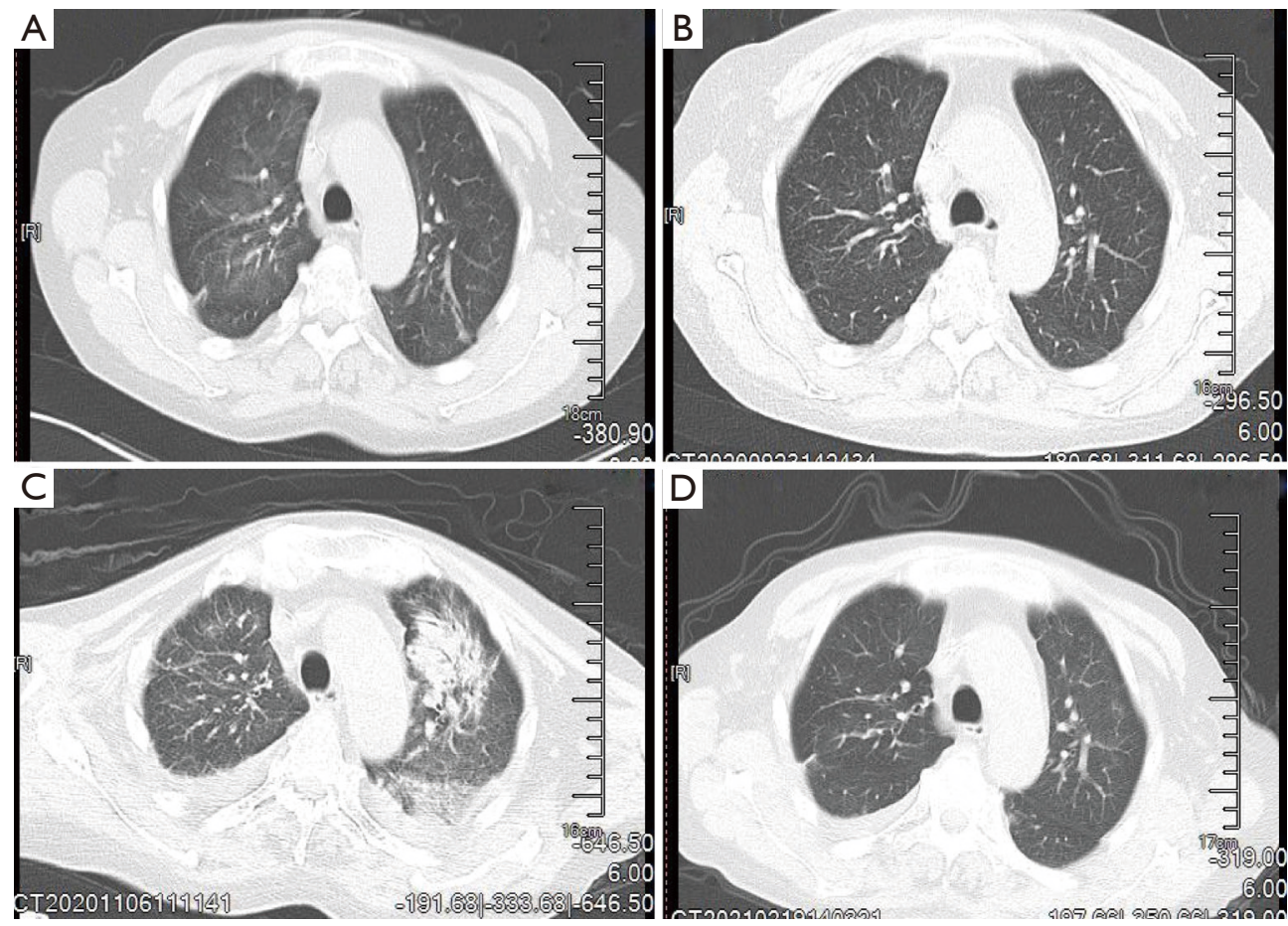

Figure 1 Radiologic presentation in Case 2. Chest CT image showed patchy ground-glass opacities in both upper lobes after 3 cycles of RTX-based immunochemotherapy (A), which significantly improved after the treatment of $80 \mathrm{mg} / \mathrm{d}$ methylprednisolone for 4 days (B). After a 3-week clinical symptom remission, the patient experienced fever and chest tightness. Further bronchoalveolar lavage fluid next-generation sequencing confirmed PCP infection. A representative CT scan revealed the diffuse patchy ground-glass opacities with an interlobular septal thickening and the presence of bilateral pleural effusion (C). The patient recovered slowly after treatment for PCP (D). CT, computed tomography; RTX, rituximab; PCP, pneumocystis pneumonia.

administration TMP/SMX in patients who have received RTX-based immunochemotherapy treatment. Such prevention may be particularly beneficial to unfit patients or patients with ILD who have received glucocorticoid treatment previously (38). For the dosage of TMP/SMX to prevent PCP, either one single strength tablet $(80 \mathrm{mg}$ TMP and $400 \mathrm{mg}$ SMX) daily or one double strength table three times a week is recommended (37). However, there is no report about when to start PCP prevention. In our practice, patients experienced lung disease after a median of three cycles of immunochemotherapy and TMP/SMX can be used for PCP prophylaxis on the third cycle of R-based immunochemotherapy.

\section{Conclusions}

In summary, this study was the first to comprehensively report that multiple blood parameters may serve as a warning for B-cell lymphoma patients with immunochemotherapyrelated ILD. Further, glucocorticoid administration and PCP prevention are crucial for B-cell lymphoma patients with immunochemotherapy-related ILD.

In addition, there were some weaknesses of this study, such as the small sample size, the lack of homogeneity in relation to the immunochemotherapy modalities and nonrandomized retrospective design. However, all reported cases in our center were included to avoid selection bias except for some undiagnosed cases. Therefore, the results of this study must be considered preliminary and should be verified by large-scale randomized controlled trials in the future.

\section{Acknowledgments}

We would like to thank all the members of the study team, the patients, and their families.

Funding: This work was supported by research grants from the National Natural Science Foundation of China (81400155, 81600114), the Jiangsu Natural Science Foundation of China (BK20140374) and the Top-Notch 
Young Health Talents, 5th Suzhou Health Professionals Program (GSWS2019035).

\section{Footnote}

Reporting Checklist: The authors have completed the STROBE reporting checklist. Available at https://dx.doi. org/10.21037/apm-21-2027

Data Sharing Statement: Available at https://dx.doi. org/10.21037/apm-21-2027

Conflicts of Interest: All authors have completed the ICMJE uniform disclosure form (available at https://dx.doi. org/10.21037/apm-21-2027). The authors have no conflicts of interest to declare.

Ethical Statement: The authors are accountable for all aspects of the work in ensuring that questions related to the accuracy or integrity of any part of the work are appropriately investigated and resolved. The study was conducted in accordance with the Declaration of Helsinki (as revised in 2013). The study was approved by the Ethics Committee of the First Affiliated Hospital of Soochow University (Suzhou, China). Individual consent for this retrospective analysis was waived.

Open Access Statement: This is an Open Access article distributed in accordance with the Creative Commons Attribution-NonCommercial-NoDerivs 4.0 International License (CC BY-NC-ND 4.0), which permits the noncommercial replication and distribution of the article with the strict proviso that no changes or edits are made and the original work is properly cited (including links to both the formal publication through the relevant DOI and the license). See: https://creativecommons.org/licenses/by-nc-nd/4.0/.

\section{References}

1. Yang Y, Zhou J, Cao C, et al. Additional possibilities of chimeric antigen receptor T-cells in B-cell lymphoma: combination therapy. Transl Cancer Res 2020;9:7310-22.

2. Pfreundschuh $\mathrm{M}$, Trümper L, Osterborg A, et al. CHOPlike chemotherapy plus rituximab versus CHOPlike chemotherapy alone in young patients with goodprognosis diffuse large-B-cell lymphoma: a randomised controlled trial by the MabThera International Trial (MInT) Group. Lancet Oncol 2006;7:379-91.
3. Pfreundschuh M, Kuhnt E, Trümper L, et al. CHOP-like chemotherapy with or without rituximab in young patients with good-prognosis diffuse large-B-cell lymphoma: 6-year results of an open-label randomised study of the MabThera International Trial (MInT) Group. Lancet Oncol 2011;12:1013-22.

4. Visco C, Quaglia FM, Bovo C, et al. Bendamustine plus rituximab: is it a BRIGHT idea? Chin Clin Oncol 2020;9:22.

5. Zelenetz AD, Gordon LI, Abramson JS, et al. NCCN guidelines insights: B-cell lymphomas, version 3.2019. J Natl Compr Canc Netw 2019;17:650-61.

6. Zhou T, Shen Q, Peng H, et al. Incidence of interstitial pneumonitis in non-Hodgkin's lymphoma patients receiving immunochemotherapy with pegylated liposomal doxorubicin and rituximab. Ann Hematol 2018;97:141-7.

7. Lim KH, Yoon HI, Kang YA, et al. Severe pulmonary adverse effects in lymphoma patients treated with cyclophosphamide, doxorubicin, vincristine, and prednisone (CHOP) regimen plus rituximab. Korean J Intern Med 2010;25:86-92.

8. Wagner SA, Mehta AC, Laber DA. Rituximab-induced interstitial lung disease. Am J Hematol 2007;82:916-9.

9. Kong H, Wang $\mathrm{Y}$, Zeng X, et al. Involvement of NLRP3 inflammasome in rituximab-induced interstitial lung disease: a case report. J Clin Pharm Ther 2014;39:691-4.

10. Mazzotta M, Giusti R, Iacono D, et al. Pulmonary fibrosis after pegylated liposomal doxorubicin in elderly patient with cutaneous angiosarcoma. Case Rep Oncol Med 2016;2016:8034832.

11. Meng L, Huang L, Xu Y, et al. Incidence of interstitial pneumonitis in breast cancer patients treated with pegylated liposomal doxorubicin. Cancer Chemother Pharmacol 2020;85:3-7.

12. Tonelli AR, Lottenberg R, Allan RW, et al. Rituximabinduced hypersensitivity pneumonitis. Respiration 2009;78:225-9.

13. Lands LC. New therapies, new concerns: rituximabassociated lung injury. Pediatr Nephrol 2010;25:1001-3.

14. Skeoch S, Weatherley N, Swift AJ, et al. Drug-induced interstitial lung disease: a systematic review. J Clin Med 2018;7:356.

15. Park SY, Kim MY, Choi WJ, et al. Pneumocystis pneumonia versus rituximab-induced interstitial lung disease in lymphoma patients receiving rituximabcontaining chemotherapy. Med Mycol 2017;55:349-57.

16. Alexandre K, Ingen-Housz-Oro S, Versini $M$, et al. Pneumocystis jirovecii pneumonia in patients treated with 
rituximab for systemic diseases: Report of 11 cases and review of the literature. Eur J Intern Med 2018;50:e23-4.

17. Hadjinicolaou AV, Nisar MK, Parfrey H, et al. Noninfectious pulmonary toxicity of rituximab: a systematic review. Rheumatology (Oxford) 2012;51:653-62.

18. Salmasi G, Li M, Sivabalasundaram V, et al. Incidence of pneumonitis in patients with non-Hodgkin lymphoma receiving chemoimmunotherapy with rituximab. Leuk Lymphoma 2015;56:1659-64.

19. Mokra D, Kosutova P. Biomarkers in acute lung injury. Respir Physiol Neurobiol 2015;209:52-8.

20. Nakamura K, Kato M, Shukuya T, et al. Surfactant protein-D predicts prognosis of interstitial lung disease induced by anticancer agents in advanced lung cancer: a case control study. BMC Cancer 2017;17:302.

21. Yamane H, Ochi N, Nagasaki Y, et al. Drug-induced interstitial lung disease in the treatment of malignant lymphoma as a potential diagnostic marker: a comparison of serum Krebs von Lungen-6 and thymus and activationregulated chemokine/CC chemokine ligand 17 . Ther Clin Risk Manag 2018;14:1457-65.

22. Chen Q, Chen DY, Xu XZ, et al. Platelet/lymphocyte, lymphocyte/monocyte, and neutrophil/lymphocyte ratios as biomarkers in patients with rheumatoid arthritis and rheumatoid arthritis-associated interstitial lung disease. Med Sci Monit 2019;25:6474-81.

23. Gao Y, Wu X, Li Y, et al. The predictive value of MLR for radiation pneumonia during radiotherapy of thoracic tumor patients. Cancer Manag Res 2020;12:8695-701.

24. Plosker GL, Figgitt DP. Rituximab: a review of its use in non-Hodgkin's lymphoma and chronic lymphocytic leukaemia. Drugs 2003;63:803-43.

25. Reckzeh B, Merte H, Pflüger KH, et al. Severe lymphocytopenia and interstitial pneumonia in patients treated with paclitaxel and simultaneous radiotherapy for non-small-cell lung cancer. J Clin Oncol 1996;14:1071-6.

26. Huang YC, Liu CJ, Liu CY, et al. Low absolute lymphocyte count and addition of rituximab confer high risk for interstitial pneumonia in patients with diffuse large B-cell lymphoma. Ann Hematol 2011;90:1145-51.

27. Du Clos TW, Mold C. C-reactive protein: an activator of innate immunity and a modulator of adaptive immunity. Immunol Res 2004;30:261-77.

28. Xu Y, Yang CS, Li YJ, et al. Predictive factors of rapidly progressive-interstitial lung disease in patients with clinically amyopathic dermatomyositis. Clin Rheumatol 2016;35:113-6.

29. Don BR, Kaysen G. Serum albumin: relationship to inflammation and nutrition. Semin Dial 2004;17:432-7.

30. Artigas A, Wernerman J, Arroyo V, et al. Role of albumin in diseases associated with severe systemic inflammation: pathophysiologic and clinical evidence in sepsis and in decompensated cirrhosis. J Crit Care 2016;33:62-70.

31. Fleck A, Raines G, Hawker F, et al. Increased vascular permeability: a major cause of hypoalbuminaemia in disease and injury. Lancet 1985;1:781-4.

32. Zhu H, Qu G, Yu H, et al. Features of $\alpha-H B D H$ in COVID-19 patients: a cohort study. J Clin Lab Anal 2021;35:e23690.

33. Yu H, Han H, Li J, et al. Alpha-hydroxybutyrate dehydrogenase as a biomarker for predicting systemic lupus erythematosus with liver injury. Int Immunopharmacol 2019;77:105922.

34. Ding J, Karp JE, Emadi A. Elevated lactate dehydrogenase $(\mathrm{LDH})$ can be a marker of immune suppression in cancer: Interplay between hematologic and solid neoplastic clones and their microenvironments. Cancer Biomark 2017;19:353-63.

35. Kubo K, Azuma A, Kanazawa M, et al. Consensus statement for the diagnosis and treatment of drug-induced lung injuries. Respir Investig 2013;51:260-77.

36. Raju S, Ghosh S, Mehta AC. Chest CT signs in pulmonary disease: a pictorial review. Chest 2017;151:1356-74.

37. Malpica L, van Duin D, Moll S. Preventing infectious complications when treating non-malignant immunemediated hematologic disorders. Am J Hematol 2019;94:1396-412.

38. Green H, Paul M, Vidal L, et al. Prophylaxis of Pneumocystis pneumonia in immunocompromised non$\mathrm{HIV}$-infected patients: systematic review and metaanalysis of randomized controlled trials. Mayo Clin Proc 2007;82:1052-9.

(English Language Editor: L. Huleatt)

Cite this article as: Xia F, Liu H, Zhang H, Ping N, Wang P, Jin Z, Zhu J, Qu C. Multiple blood parameters may serve as a warning to immunochemotherapy-related interstitial lung disease in B-cell lymphoma. Ann Palliat Med 2021;10(9):96609668. doi: 10.21037/apm-21-2027 